\title{
Energy-Saving Research on Residential Gas Heating System in Cold Area Based on System Dynamics
}

\author{
Yingchun $\mathrm{Cao}^{1}$, Jie Yang ${ }^{1}$, Jiwei $\mathrm{Li}^{2 *}$ \\ ${ }^{1}$ School of Architecture and Art, Hebei University of Architecture, Zhangjiakou 075000, China \\ ${ }^{2}$ College of Civil Engineering and Architecture, Hebei University, Baoding 071002, China
}

Corresponding Author Email: 1014206030@tju.edu.cn

https://doi.org/10.18280/ijht.380222

Received: 18 December 2019

Accepted: 27 March 2020

\section{Keywords:}

system dynamics, wall-hung boiler, heating,

energy saving

\begin{abstract}
In recent years, northern China has continuously promoted gas-fired heating systems, and more residential heating terminals have adopted the gas wall-hung boiler systems. Factors such as the output power of the wall-hung boiler, the heating operation mode, and the thermal insulation performance of the building envelope all affect the final building energy consumption. The authors attempt to achieve the goal of energy saving through systematical analysis of these factors. The method of system dynamics analysis was introduced into the energy consumption analysis of wall-hung boiler. Besides, taking a residential building in Tianjin as an example, a system dynamic model of the wall-hung boiler system was constructed and simulated; the simulation analysis was conducted about the impact of the thermal insulation performance of the enclosure structure on the operation and output power of the wall-hung boiler, the impact of the rated power on the energy consumption of the building, and the impact of the temperature difference at shutdown on the energy consumption of the building, and the impact of the temperature difference at shutdown on the energy consumption of the building. The results showed that with the insulation layer thickness of $100 \mathrm{~mm}$, the energy-saving effect is better; with the wall-hung boiler output power of $20 \mathrm{KW}$, the average energy consumption of the building is relatively small; the low system temperature difference at shutdown is more conducive to system energy saving.
\end{abstract}

\section{INTRODUCTION}

The proportion of heating energy consumption in the total social energy consumption in northern China has been increasing. At the end of 2016, the heating area in the northern region had reached 20.6 billion square meters, of which $11 \%$, about 2.2 billion square meters of buildings, use natural gas as heating energy [1] According to the Clean Heating Plan for the Northern Region in Winter, it's estimated that by 2021, 1.8 billion square meters of natural gas heating area will be added in the northern region, with a cumulative increase of 23 billion cubic meters of gas consumption [2]. Household gas wallhung boilers are suitable for areas that are not covered by the heating network. Because of their low cost, simple installation method and easy household control, they have become one of the main gas heating methods in cold areas in winter [3]. Meanwhile, the building energy conservation movement has been constantly carried out in China. But the residents are sensitive to heating costs. It has become a research hotspot on how to ensure the heating effect while maximizing energy conservation now [4].

At present, many scholars have carried out related research on the heating problem of residential buildings. BeausoleilMorrison et al. [5] studied solar heating of cold-climate households and found that seasonal energy storage can solve the heating problem of residential buildings. Alptekin et al. [6] conducted research on residential heating systems with natural gas thermal power, concluding that such heating systems can reduce power consumption and have better economic efficiency. O'Rear et al. [7] performed a comparative analysis between natural gas heating and electric heating systems for low-energy residential buildings, and discovered that electric heating systems have better economics, while natural gas heating systems have less environmental impact and have less impact on the environment, which is more favorable for sustained development. Thomas et al. [8] analyzed the types of envelope structure from the perspective of steady-state heat flow and dynamic heat flow, and proposed that different types of heating systems should choose appropriate envelope structure systems. Evin and Ucar [9] carried out the research on the heating and cooling load of residential buildings in Turkey, and compared the effects of different insulation materials on the comprehensive energy consumption of buildings. Zanetti et al. [10] studied the energy saving of photovoltaic heat pumps for optimally controlled hybrid building heating systems and found that heating systems with optimal energy management strategies can save up to $20 \%$ of energy costs.

Regarding wall-hung boiler systems, some scholars have also performed a series of studies. Mattinen et al. [11] analyzed the independent heating system of gas wall-hung boilers and air source heat pumps, finding that gas wall-hung boiler systems are more suitable for northern residential heating; Ekholm et al. [12] analyzed the direct relationship between indoor temperature and energy consumption of household gas wall-hung boilers through the experiments; 
Rodríguez-Pertuz et al. [13] concluded in their study that compared with central heating systems, wall-hung boilers have the characteristics of autonomous control, flexible installation, and high comfort adaptability. Generally speaking, most of the current researches focus on the applicability of wall-hung boilers, but there are relatively few studies on the systemic energy saving of wall-hung boilers. As an independent heating system, the heating and energy saving of wall-hung boiler is subjected to many factors, including the power of the wall-hung boiler, the heating operation mode, and the insulation performance of the building envelope etc. [14]. These factors interact and influence each other. It is difficult to analyze them using a single method. System dynamics analysis is a currently widely used tool in ecosystems, mechanical systems, management systems and other fields [15-18]. It can analyze the operation of complex systems under different operating conditions [19], and is suitable for the research on the effect of system heating in the gas wall-hung boilers. Through analysis for these factors using system dynamics tools, this paper aims to obtain the best operating parameters of the wall-hung boiler heating system and achieve system energy saving.

\section{MATERIAL AND METHODS}

\subsection{Operation mode of wall-hung boiler system}

Residential wall-hung boiler is an automatic independent heating system that uses natural gas as energy and water as heat medium to increase water temperature through gas combustion, thereby meeting residential heating requirements [20]. It consists of combustion system, heat exchange system, waterway system and control system. The combustion system and the water system start simultaneously after sensing the heating demand through the boiler's temperature sensing system. Then, the heat exchange system and the combustion chamber burn gas for heat exchange, and the heated hot water is circulated through the circulating water pump. When the hot water is delivered to the radiator, the radiator supplements the heat to the room for maintaining the indoor temperature. With the water temperature or room temperature reaching the set temperature, the system temporarily stops running and maintains the room temperature by the heat accumulated in the room; with the room temperature or water temperature lower than the set temperature, the system restarts. In this way, the heating work is completed.

\subsection{Advantages of system dynamics method during its} application in energy consumption analysis of wall-hung boiler

Currently, most of the researches on heating energy saving are based on the first law of thermodynamics, using the heat balance equation [21], or energy consumption simulation software such as EnergyPlus, and eQUEST, etc. [22]. These methods are suitable for heating with a relatively stable heat source [23]. The wall-hung boiler system needs to be started frequently, and the energy fluctuates frequently. The traditional simulation method cannot accurately simulate the building energy consumption of this system. Moreover, factors such as the output power of the system, the heating operation mode, and the insulation performance of the building envelope affect the overall property of energy saving; the mechanism of action between different factors is relatively complex, which is difficult to explain with a single mathematical model. Thus, it's necessary to find a new analysis tool or method for the energy-saving problem of wallhung boiler system.

As a comprehensive research method, system dynamics is mainly used to study complex systems, and solve systemic problems by studying the feedback and operation of system information [24]. It is suitable for studying dynamic systems, and constructing dynamic system models through analysis of the internal organizational structure, material flow, information flow, and feedback mechanism of the system. It is based on system theory and integrates cybernetics and information pathways, which can solve and analyze system operation problems in which multiple factors influence each other [25]. Therefore, the analysis method of system dynamics can well solve the energy consumption problem of the wallhung boiler heating operation.

When applying system dynamics to solve practical problems, it is first necessary to analyze the constituent elements and specific parameters of the system and find the operation mode, then to establish the system process according to the system operation law and run the model simulation in the software, and finally perform the results analysis.

\subsection{Setting the elements of wall-hung boiler system}

\subsubsection{Case selection and system element analysis}

A residential unit in Tianjin was selected as an example, and its system dynamic model was constructed for specific analysis, as shown in Figure 1.

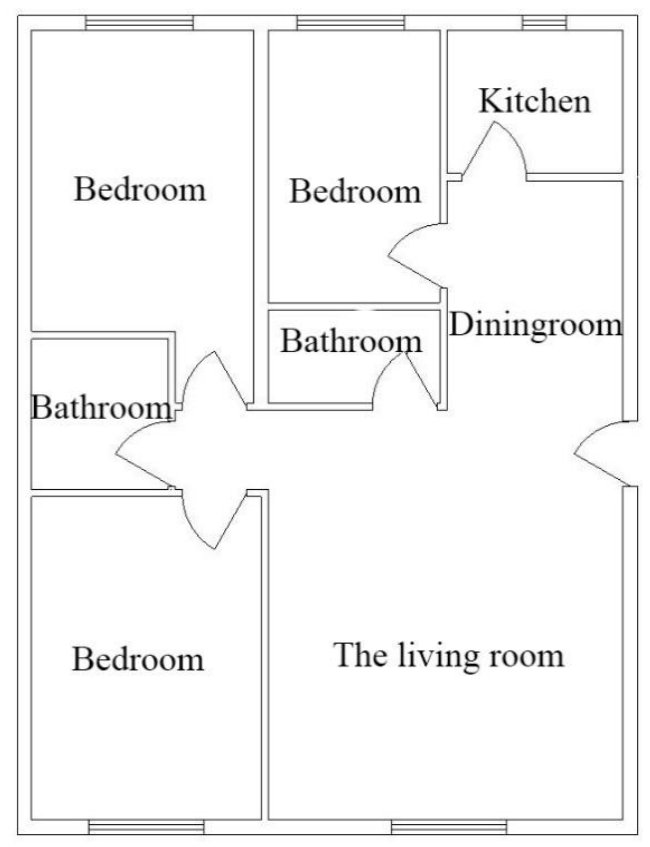

Figure 1. A high-rise residential unit type in Tianjin

This was an intermediate unit and adopted a shear wall structure with the building area of $98.61 \mathrm{~m}^{2}$, the building height of $2.9 \mathrm{~m}$, the exterior wall area $S_{\mathrm{w}}$ of $40.20 \mathrm{~m}_{2}$, the exterior window area $S_{c}$ of $11.70 \mathrm{~m}^{2}$, and the heat transfer coefficient $\mathrm{K}_{\mathrm{c}}$ of $2.3 \mathrm{~W} /\left(\mathrm{m}^{2} \bullet \mathrm{K}\right)$. The exterior wall was constructed from the inside to the outside as follows: lime-painted, 20mm thick; thermal conductivity $\lambda_{1}$ for $0.81 \mathrm{~W} /(\mathrm{m} \cdot \mathrm{K})$; reinforced concrete shear wall, $200 \mathrm{~mm}$ thick; thermal conductivity $\lambda_{2}$ for 1.74 
$\mathrm{W} /(\mathrm{m} \cdot \mathrm{K})$; expanded polystyrene as a thermal insulation material, and a thermal conductivity $\lambda_{3}$ of $0.041 \mathrm{~W} /(\mathrm{m} \cdot \mathrm{K})$; a decorative surface layer of cement mortar, $20 \mathrm{~mm}$ thick, with a thermal conductivity $\lambda_{4}$ of $0.93 \mathrm{~W} /(\mathrm{m} \bullet \mathrm{K})$. In addition, the total thermal resistance of the outer wall was $\mathrm{R}_{\mathrm{w}}$, the heat transfer coefficient of the outer wall was $\mathrm{K}_{\mathrm{w}}$, and the overall heat dissipation strength of the enclosure structure was Q. Q was affected by the indoor and outdoor temperature difference and the heat transfer coefficient of the enclosure structure. When the heat transfer coefficient of the enclosure structure is constant, the greater the indoor and outdoor temperature difference, the higher the overall heat dissipation strength of the enclosure structure; when the indoor and outdoor temperature difference is constant, the greater the heat transfer coefficient of the envelope structure, the higher the overall heat dissipation strength of the envelope structure. Thus, more energy E needs to be supplemented by the wall-hung boiler system. Also, factors such as the power $\mathrm{P}$ and the temperature difference $\mathrm{T}$ at shutdown will also affect the heat dissipation of the enclosure structure. These factors need to be comprehensively considered when analyzing energy consumption.

\subsubsection{System energy-balance equation}

The entire system follows the law of conservation of energy. In order to maintain a relatively constant indoor temperature, the heat added to the room by the wall-hung boiler needs to be equal to the heat lost by the enclosure structure:

$$
E=Q \times t
$$

The basic system dynamics model of indoor energy consumption was constructed according to the above parameters and relations.

\subsection{Establishing the system dynamic model of wall-hung boiler system}

\subsubsection{Model establishment and flow chart design of wall-hung} boiler system

To establish a wall-hung boiler system model, the operation law of different subsystems and the relationship between the subsystems under the principle of energy balance need to be analyzed to find the main contradiction, and determine the relationship between the different subsystems. Then, the elements of the subsystems should be confirmed, and assigned with values based on actual data or theory.

According to the operating principle of the wall-hung boiler system, the system flow chart was established in the system power software Vensim Ple, as shown in Figure 2. This process mainly consists of two subsystems: the energy supply subsystem and temperature control subsystem. After the system is started, the temperature control subsystem first detects and monitors the indoor temperature through the monitor 2. When the indoor temperature is lower than the set temperature, the energy supply subsystem is started, then the gas boiler works, and the temperature of the heat transfer medium in the radiator rises, supplying heat to the room. Then, monitor 1 starts to monitor the temperature of the heat transfer medium. When the temperature of the heat transfer medium is higher than the set shutdown temperature and the indoor temperature is higher than the set minimum room temperature, the energy supply system is shut down through the controller. At this time, energy flows outward through the enclosure structure, and the temperature of the heat transfer medium begins to drop. When the temperature drop of the heat transfer medium is greater than the temperature difference at shutdown and the room temperature is lower than the set minimum temperature, the heating system is turned on and the wall-hung boiler starts to work. This shall provide heat to the room and maintain a relatively stable indoor temperature.

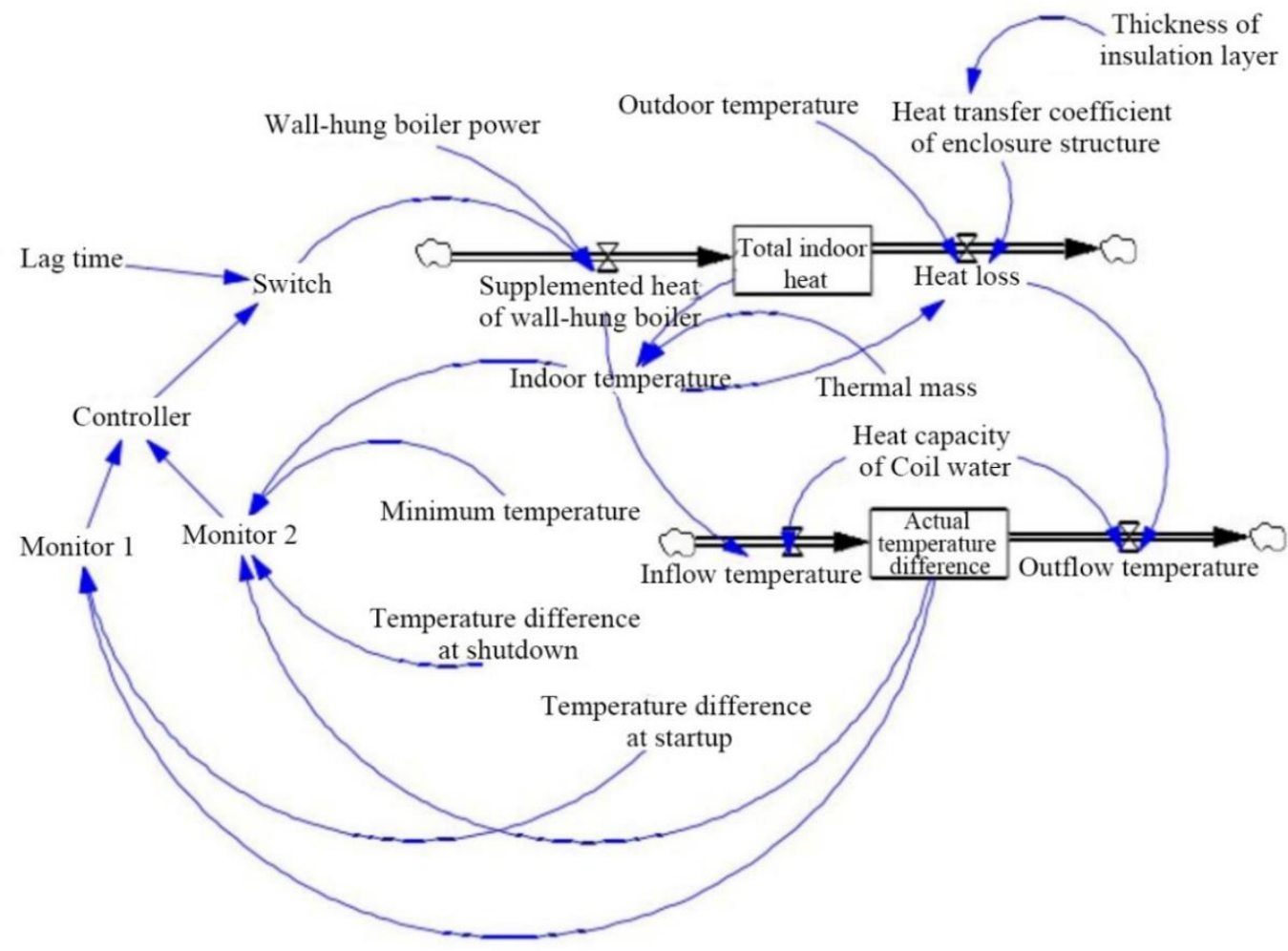

Figure 2. System dynamics flow chart of the wall-hung boiler system 
2.4.2 Setting the heating operation system of wall-hung boiler The weather conditions of Tianjin in the cold area were taken as the external boundary, and the outdoor calculated temperature during the heating period was selected as the external environment calculation parameter of the system. The system is divided into an energy subsystem and a control subsystem. The energy subsystem mainly include: wall-hung boiler output power, heat dissipation of the enclosure structure and heat transfer coefficient of the enclosure structure; the control subsystem mainly includes: monitor 1, monitor 2, controller, switch, temperature difference at shutdown, temperature difference at start-up, minimum room temperature, actual temperature difference, increased temperature, and decreased temperature, etc; the outdoor calculated temperature is set to $-9^{\circ} \mathrm{C}$, the indoor calculated temperature is $20^{\circ} \mathrm{C}$, and the simulation duration is 96 hours. According to the previous analysis, the variables that ultimately affect energy consumption include the thermal insulation performance of the enclosure structure, the output power of the wall-hung boiler, and the temperature difference at shutdown. The simulation analysis for these factors was performed in the following section.

\section{RESULTS AND DISCUSSION}

3.1 Analysis for the impact of the thermal insulation performance of the envelope structure on the operation of the wall-hung boiler

Adjusting the thickness of the insulation layer can change the heat transfer coefficient of the envelope structure, directly affect the energy consumption of the building, and further influence the operating law of the wall-hung boiler. The thickness of the insulation layer was adjusted to $50 \mathrm{~mm}$, $100 \mathrm{~mm}, 150 \mathrm{~mm}$, and $200 \mathrm{~mm}$, respectively, as shown in Figure 3. It can be seen that with the thickness of the insulation layer increasing, the start-up time interval of the wall-hung boiler system increases, and the running time decreases.

The total running time is shown in Figure 4. When the thickness of the insulation layer was $50 \mathrm{~mm}$, the running time was up to $65 \mathrm{~h}$, about $68 \%$ of the time was in operation, and the energy output was higher. With the thickness of the insulation layer increasing, the system running time was gradually shortened. When the thickness of the insulation layer was increased to $200 \mathrm{~mm}$, the total operating time was shortened to 18.5 hours, and only about $19 \%$ of the time was in the operating state, which greatly shortened the operating time, and reduced the energy output.

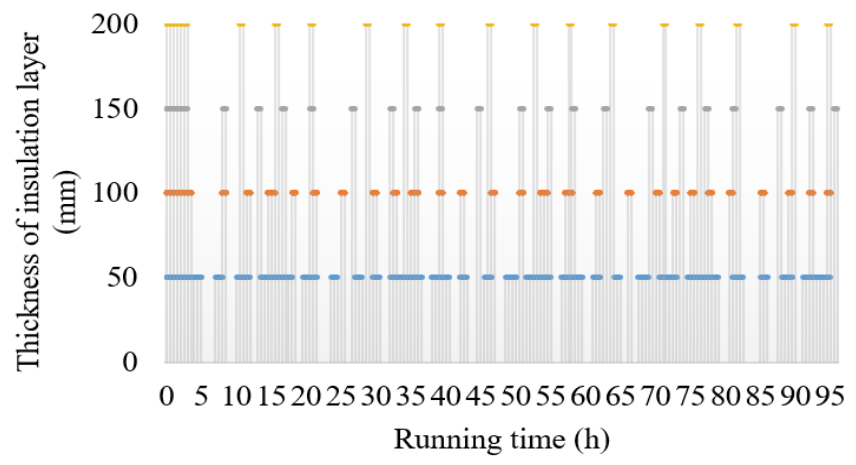

Figure 3. Heating start-stop time of wall-hung boiler
Total running time of wall-hung boiler

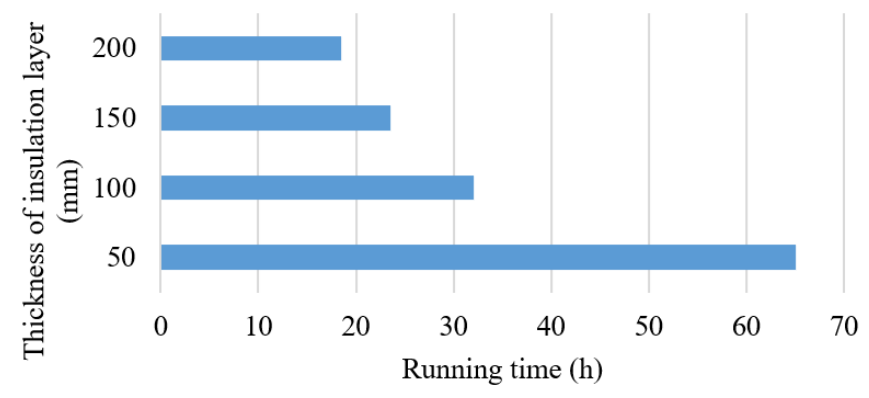

Figure 4. Boiler running time

3.2 The impact of the insulation layer thickness of the envelope structure on the average output power of the wall-hung boiler

Setting the step length of the insulation layer thickness change of the envelope structure to $10 \mathrm{~mm}$, the initial value to $40 \mathrm{~mm}$, and the maximum value to $200 \mathrm{~mm}$, the simulation was performed to obtain the average output power curve of the wall-hung boiler, as shown in Figure 5. It can be found that with the insulation layer thickness increasing, the average output power of the wall-hung boiler becomes smaller, that is, the lower the overall energy consumption, the more the overall trend conforms to the logarithmic distribution law. When the thickness of the enclosure structure is $40 \mathrm{~mm}$, the average output power of the wall-hung boiler is $23.72 \mathrm{KW}$; when it increases to $200 \mathrm{~mm}$, the output power is $6.74 \mathrm{KW}$. When the thickness of the insulation layer increases from $40 \mathrm{~mm}$ to $100 \mathrm{~mm}$, the overall thickness increases by $60 \mathrm{~mm}$, and the average power of the wall-hung boiler decreases by $12 \mathrm{KW}$; when the insulation thickness increases from $100 \mathrm{~mm}$ to $200 \mathrm{~mm}$, the overall thickness increases by $100 \mathrm{~m}$, and the output power of the wall-hung boiler decreases by $5 \mathrm{KW}$. Thus, it is more economical to increase the insulation layer from $40 \mathrm{~mm}$ to $100 \mathrm{~mm}$, and the further increase of thickness shall reduce the energy saving efficiency of the insulation layer.

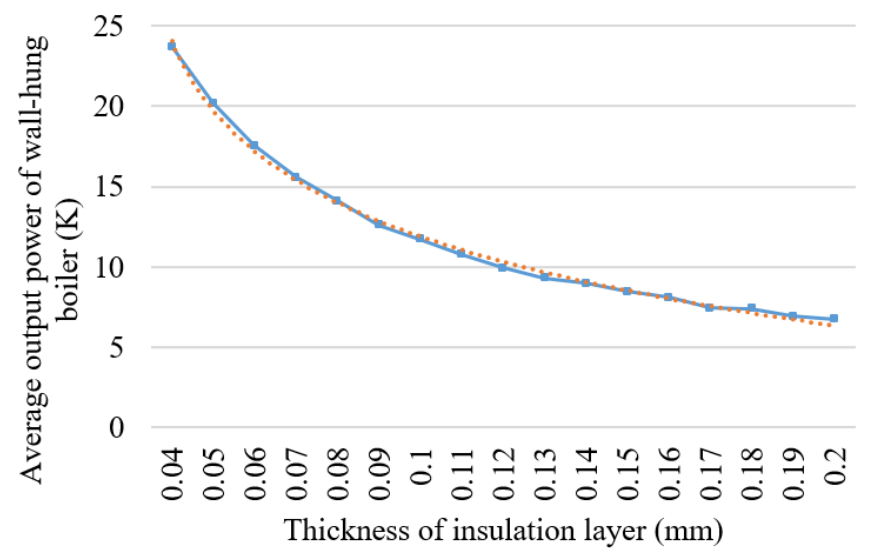

Figure 5. The impact of insulation thickness on average output power of wall-hung boiler

\subsection{The impact of wall-hung boiler rated power on energy consumption}

The rated power of the wall-hung boiler is usually $16 \mathrm{KW}$, $18 \mathrm{KW}, 20 \mathrm{KW}, 24 \mathrm{KW}, 28 \mathrm{KW}, 32 \mathrm{KW}$, and $40 \mathrm{KW}$. The previous analysis shows that when the rated power is low, the 
wall-hung boiler has a longer working time; when the rated power is increased, working hours will be shortened. Generally speaking, an increase in working time will reduce the service life of wall-hung boilers, while an increase in rated power will increase the overall energy consumption of the building. Thus, it's necessary to select a reasonable rated output power. By simulating several commonly used wallhung boilers (Figure 6), it can be found that when the rated power is $20 \mathrm{KW}$, the average energy consumption of the building is relatively lower, and the system running time is relatively less, which can be the best choice for this project.

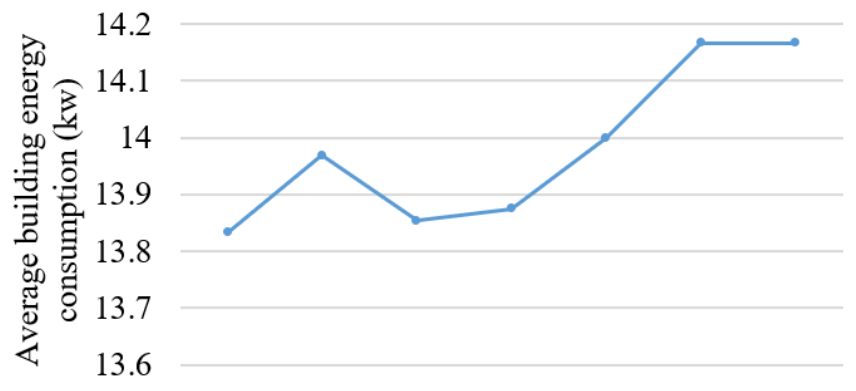

16KW 18KW 20KW 24KW 28KW 32KW 40KW

Rated power of wall-hung boiler

Figure 6. The impact of wall-hung boiler power on average building energy consumption

\subsection{The impact of temperature difference at shutdown on building energy consumption}

Another important parameter of the wall-hung boiler system is the temperature difference at shutdown. The shutdown of the wall-hung boiler is controlled by setting the temperature difference, that is, when the heat transfer medium is higher than a certain value of the set room temperature, the system will automatically shut down; usually the setting range is $5^{\circ} \mathrm{C}$ to $20^{\circ} \mathrm{C}$. Figure 7 shows that as the temperature difference at shutdown increases, the average energy consumption of the building gradually increases. This is mainly because with the temperature difference at shutdown increasing, the temperature of the heat transfer medium shall rise higher, and the indoor temperature will also increase accordingly. Then, the indoor and outdoor temperature difference and heat loss will further increase. Therefore, setting a relatively low temperature difference at shutdown is more conducive to energy saving.

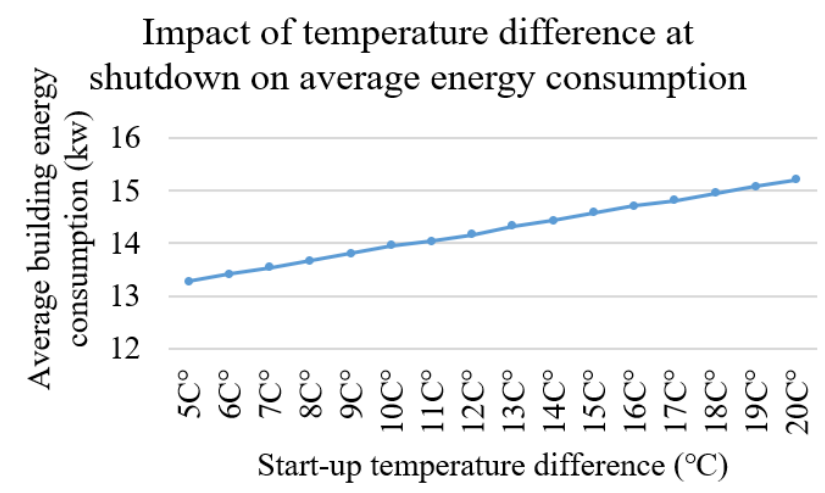

Figure 7. The influence of the temperature difference at shutdown on the average energy consumption

\section{CONCLUSIONS}

The study found that the use of system dynamics can help to easily analyze the impact of different parameters on building energy consumption when analyzing the energy saving of wall-hung boiler systems in cold areas, because it is beneficial to the determination of parameters and the improvement of design schemes. In general, the improvement in the thermal insulation performance of the enclosure structure is improved, then the starting frequency of the wallhung boiler will decrease, the running time of a single startup will be greatly reduced, the average output power of the wallhung boiler will decrease, and the energy consumption of the building will be reduced. Through the case analysis, it can be seen that when the thickness of the insulation layer of the enclosure structure is increased from $40 \mathrm{~mm}$ to $100 \mathrm{~mm}$, the overall energy consumption will decrease significantly; the rated power of the wall-hung boiler also has a certain impact on the energy consumption of the building, i.e., when the rated power of the wall-hung boiler is $20 \mathrm{KW}$, the average energy consumption of the building is relatively small, and the continuous working time is relatively short; the relatively low temperature difference at shutdown is more conducive to energy saving, because the higher shutdown temperature difference setting can result in a greater indoor and outdoor temperature difference, and the increase in heat loss.

\section{ACKNOWLEDGMENT}

This research was funded by Natural Science Foundation of Hebei Province of China (Grant No.: E2019201230), Hebei Education Department (Grant No.: QN2019187), High-level Talent Research Startup Project of Hebei University (Grant No.: 521000981386), National Natural Science Foundation of China (Grant No.: 51708394, 51708378).

\section{REFERENCES}

[1] Mao, X.K., Wang, L.X., Li, J.W., Quan, X.L., Wu, T.Y. (2019). Comparison of regression models for estimation of carbon emissions during building's lifecycle using designing factors: A case study of residential buildings in Tianjin, China. Energy and Buildings, 204(12): 109519. https://doi.org/10.1016/j.enbuild.2019.109519

[2] Fen, C.Y., Duan, Z.F., Sun, W.Y. (2018). Impact of promoting clean heating in northern China on natural gas market. China Energy, 40(6): 28-30, 37. https://doi.org/10.3969/j.issn.1003-2355.2018.06.005

[3] Izadyar, N., Ghadamian, H., Ong, H.C., Tong, C.W., Shamshirband, S. (2015). Appraisal of the support vector machine to forecast residential heating demand for the District Heating System based on the monthly overall natural gas consumption. Energy, 93: 1558-1567. https://doi.org/10.1016/j.energy.2015.10.015

[4] Sanaye, S., Ghafurian, M.M. (2016). Applying relative equivalent uniform annual benefit for optimum selection of a gas engine combined cooling, heating and power system for residential buildings. Energy and Buildings, 128: 809-818.

https://doi.org/10.1016/j.enbuild.2016.07.015

[5] Beausoleil-Morrison, I., Kemery, B., Wills, A.D., Meister, C. (2019). Design and simulated performance of 
a solar-thermal system employing seasonal storage for providing the majority of space heating and domestic hot water heating needs to a single-family house in a cold climate. Solar Energy, 191: 57-69. https://doi.org/10.1016/j.solener.2019.08.034

[6] Alptekin, M., Calisir, T., Baskaya, S. (2017). Design and experimental investigation of a thermoelectric selfpowered heating system. Energy Conversion and Management, 146: 244-252.

https://doi.org/10.1016/j.enconman.2017.05.033

[7] O'Rear, E., Webb, D., Kneifel, J., O'Fallon, C. (2019). Gas vs electric: Heating system fuel source implications on low-energy single-family dwelling sustainability performance. Journal of Building Engineering, 25: 100779. https://doi.org/10.1016/j.jobe.2019.100779

[8] Thomas, L.P., Marino, B.M., Muñoz, N. (2020). Steadystate and time-dependent heat fluxes through building envelope walls: A quantitative analysis to determine their relative significance all year round. Journal of Building Engineering, 29:

101122 . https://doi.org/10.1016/j.jobe.2019.101122

[9] Evin, D., Ucar, A. (2019). Energy impact and ecoefficiency of the envelope insulation in residential buildings in Turkey. Applied Thermal Engineering, 154: 573-584.

https://doi.org/10.1016/j.applthermaleng.2019.03.102

[10] Zanetti, E., Aprile, M., Kum, D., Scoccia, R., Motta, M. (2020). Energy saving potentials of a photovoltaic assisted heat pump for hybrid building heating system via optimal control. Journal of Building Engineering, 27: 100854. https://doi.org/10.1016/j.jobe.2019.100854

[11] Mattinen, M.K., Nissinen, A., Hyysalo, S., Juntunen, J.K. (2015). Energy use and greenhouse gas emissions of airsource heat pump and innovative ground-source air heat pump in a cold climate. Journal of Industrial Ecology, 19(1): 61-70. https://doi.org/10.1111/jiec.12166

[12] Ekholm, T., Karvosenoja, N., Tissari, J., Sokka, L., Kupiainen, K., Sippula, O., Savolainen, I. (2014). A multi-criteria analysis of climate, health and acidification impacts due to greenhouse gases and air pollution - the case of household-level heating technologies. Energy Policy, 74: 499-509. https://doi.org/10.1016/j.enpol.2014.07.002

[13] Rodríguez-Pertuz, M.L., Terés-Zubiaga, J., CamposCelador, A., González-Pino, I. (2020). Feasibility of zonal space heating controls in residential buildings in temperate climates: Energy and economic potentials in Spain. Energy and Buildings, 218: 110006 https://doi.org/10.1016/j.enbuild.2020.110006

[14] Nazari-Heris, M., Mohammadi-Ivatloo, B., Asadi, S. (2020). Optimal operation of multi-carrier energy networks with gas, power, heating, and water energy sources considering different energy storage technologies. Journal of Energy Storage, 31: 101574. https://doi.org/10.1016/j.est.2020.101574

[15] Sayyadi, R., Awasthi, A. (2017). A system dynamics based simulation model to evaluate regulatory policies for sustainable transportation planning. International
Journal of Modelling and Simulation, 37(1): 25-35. https://doi.org/10.1080/02286203.2016.1219806

[16] Agyapong-Kodua, K., Weston, R.H., Ratchev, S. (2012). The integrated use of enterprise and system dynamics modelling techniques in support of business decisions. Advances in Decision Sciences, (1): 6483-6491. http://dx.doi.org/10.1155/2012/804324

[17] Li, J.W., Cao, Y.C., Zhu, Y.Q., Xu, C., Wang, L.X. (2019). System dynamic analysis of greenhouse effect based on carbon cycle and prediction of carbon emissions. Applied Ecology and Environmental Research, 17(2): 5067-5080.

http://dx.doi.org/10.15666/aeer/1702 50675080

[18] Abdulrahman, I., Radman, G. (2019). Simulink-based programs for power system dynamic analysis. Electrical Engineering, 101(2): 345-356. http://dx.doi.org/10.1007/s00202-019-00781-1

[19] Rouwette, E.A., Größler, A., Vennix, J.A. (2004). Exploring influencing factors on rationality: A literature review of dynamic decision-making studies in system dynamics. Systems Research and Behavioral Science: The Official Journal of the International Federation for Systems Research, 21(4): 351-370. https://doi.org/10.1002/sres.647

[20] Iwaro, J., Mwasha, A. (2019). Effects of using coconut fiber-insulated masonry walls to achieve energy efficiency and thermal comfort in residential dwellings. Journal of Architectural Engineering, 25(1): 04019001. https://doi.org/10.1061/(ASCE)AE.1943-5568.0000341

[21] Natephra, W., Yabuki, N., Fukuda, T. (2018). Optimizing the evaluation of building envelope design for thermal performance using a BIM-based overall thermal transfer value calculation. Building and Environment, 136: 128-145. https://doi.org/10.1016/j.buildenv.2018.03.032

[22] Fumo, N., Mago, P., Luck, R. (2010). Methodology to estimate building energy consumption using EnergyPlus Benchmark Models. Energy and Buildings, 42(12): 2331-2337. https://doi.org/10.1016/j.enbuild.2010.07.027

[23] Lopes, M.A., Antunes, C.H., Reis, A., Martins, N. (2017). Estimating energy savings from behaviours using building performance simulations. Building Research \& Information, $\quad 45(3)$ https://doi.org/10.1080/09613218.2016.1140000

[24] Mora, M., Cervantes-Pérez, F., Gelman-Muravchik, O., Forgionne, G.A. (2011). Modeling the strategic process of decision-making support systems implementations: A system dynamics approach review. IEEE Transactions on Systems, Man, and Cybernetics, Part C (Applications and Reviews), 42(6): 899-912. https://doi.org/10.1109/TSMCC.2011.2171482

[25] Zhao, B., Tang, T., Ning, B. (2017). System dynamics approach for modelling the variation of organizational factors for risk control in automatic metro. Safety Science, $\quad 94: \quad 128-142$. https://doi.org/10.1016/j.ssci.2017.01.002 